\title{
Análisis del contacto precoz de los estudiantes de medicina con el sistema sanitario
}

\author{
Ramón Miralles, Mariano Sentí, Luisa García, Jesús Escribano, Nuria Orfila, Josep E. Baños
}

Introducción. Se presentan los resultados de una experiencia sobre contacto precoz con la realidad asistencial en forma de una asignatura denominada 'Prácticas de Grado', que se cursa en primer, segundo y tercer curso del grado conjunto de medicina de la Universitat Autònoma de Barcelona y de la Universitat Pompeu Fabra.

Sujetos y métodos. Se solicitó a los estudiantes que habían cursado las asignaturas en primer, segundo o tercer curso su opinión voluntaria y anónima sobre la asunción de sus principales objetivos y competencias, y su grado de satisfacción sobre los aspectos generales y de organización. Se realizó un análisis comparativo por cursos y por la importancia de la figura del tutor (médico/a o enfermera/o) en cada una de las situaciones.

Resultados. Participaron 124 estudiantes. Globalmente, una amplia mayoría consideraba que los objetivos educativos y las competencias se habían alcanzado de forma satisfactoria. Según el ítem valorado, las respuestas 'completamente de acuerdo' y 'de acuerdo' oscilaron entre el $60,5 \%$ y el $94,4 \%$. No se observaron diferencias significativas entre cursos, excepto en la pregunta referida a la competencia de modelos de relación clínica, donde los estudiantes de tercero dieron valores inferiores $(p<0,05)$. Respecto al grado de satisfacción, fue elevado en general (rango de medias: 7,03-8,28), excepto en el ítem de 'horario'. Los estudiantes de tercer curso puntuaron de forma inferior respecto a los ítems 'atención de los tutores' $(p<0,01)$, 'aprendizaje' $(p<0,01)$ y 'experiencia vital' $(p<0,01)$. Respecto a los tutores, no se observaron diferencias significativas en ninguno de los factores, excepto en 'comprensión de otras profesiones sanitarias'.

Conclusiones. Se concluye que los estudiantes que cursaron las asignaturas mostraron una opinión favorable y una satisfacción razonable, sin grandes diferencias dependiendo del curso o del tutor asignado.

Palabras clave. Competencias transversales. Contacto precoz. Educación médica. Evaluación. Profesionalismo.

\section{An analysis of early contact of medical students with health care system}

Introduction. The paper reports the results of an experience considering the early contact of medical students with medical care in the joint degree of Medicine of Universitat Autònoma de Barcelona and Universitat Pompeu Fabra. Students follow the subject 'Prácticas de Grado' in the first, second and third academic year.

Subjects and methods. Students who had completed the subject in first, second and/or third year were asked to complete voluntarily and anonymously a questionnaire about their opinion on how educative objectives and competences were achieved, as well as on their satisfaction of general and organizational aspects of the subjects. Later, a comparative analysis was performed considering the students' scores by academic year and tutor (physician or nurse).

Results. One hundred and twenty four students participated. Most of them considered that educative objectives and competences were achieved. Considering each item, the answers 'I fully agree' and 'I agree' ranged from 60.5\% to $94.4 \%$. No differences were observed among students of different years, except in the question regarding the models of clinical relationship where third-year students gave the smallest values $(p<0.05)$. They were also highly satisfied with general and organizational aspects (range of means: 7.03-8.28), except in 'timetable' item (6.25). Third-year students scored higher than their peers the items 'tutor supervision' $(p<0.01)$, 'learning' $(p<0.01)$ and 'vital experience' $(p<0.01)$. Regarding the comparisons by tutors, no differences were seen with the exception of 'understanding of other health professionals'.

Conclusions. It is concluded that students who completed the subjects reported high scores regarding their opinion of the reaching the educational objectives and competences. No great differences were seen when compared by academic year or tutor.

Key words. Communication skills. Early contact. Evaluation. Generic competences. Medical education. Professionalism.
Departamento de Medicina; Unidad Docente del Parc de Salut Mar; Facultad de Medicina; Universitat Autònoma de Barcelona (R. Miralles). Departamento de Ciencias Experimentales y de la Salud; Facultad de Ciencias de la Salud y de la Vida; Universitat Pompeu Fabra (M. Sentí, J.E. Baños). Unidad de Formación Continuada del Parc de Salut Mar (L. García, J. Escribano, N. Orfila). Barcelona, España.

Correspondencia:

Dr. Josep Eladi Baños Díez. Departamento de Ciencias Experimentales y de la Salud. Universitat Pompeu Fabra. Parc de Recerca Biomèdica de Barcelona. Doctor Aiguader, 88. E-08003

Barcelona.

Fax:

+34933160865

E-mail:

josepeladi.banos@upf.edu

Agradecimientos:

F. Blasco, J. Cánovas, E. del Cotillo, S. Garcia, C. Farriols (Hospital de I'Esperança); S. Cordero, C. Crespo, M.M. Forner, C. Hernández, C. Hidalgo, M.J. Ligori, L. Martínez, P. Navarro (Hospital del Mar); A. Boix, N. Calderón, M. Hernández, A. Mané, C. Masip, M.J. Robles, C. Roqueta, A. Rodríguez, N. Saavedra,

G. Vallecillo, S. Ubero (Centre Fòrum); L. Crespo, V. Iglesias, N. López (Centre Emili Mira); A. Bassa, A. Clara, A. Gurt, O. Peral, M.L. Pérez (CAP Vila Olímpica); N. Amorós, X. Rodríguez, M.J. Ribó, J.M. Verdú (CAP Sant Martí); M. Anoro, P. Sancho, M. Sánchez (CAP Besòs); A. Blanch, S. Moreno, M. Porta, S. Rivas (CAP Larrard); J.M. Bellmunt,

F. Barbosa, C.L. López, C. Izard,

E. Tarradas (CAP Barceloneta).

Conflicto de intereses: No declarado.

Competing interests: None declared.

(c) 2015 FEM 


\section{Introducción}

Durante muchos años, los planes de estudio de los estudiantes de medicina se han caracterizado por la existencia de una marcada separación entre los denominados ciclo preclínico y clínico [1]. Con ello, el contacto con el sistema sanitario se postergaba con frecuencia al tercer curso, en el que existían algunas asignaturas de carácter clínico, pero en el que aún tenían un papel fundamental las llamadas 'asignaturas básicas'. Sin desmerecerlas, esta situación tenía diversos inconvenientes. En primer lugar, descontextualizaba algunos de los conocimientos obtenidos en dichas asignaturas básicas de los adquiridos en las asignaturas clínicas, con lo que los estudiantes podían tener dificultades en comprender su gran utilidad [2]. En segundo, retrasaba casi a la mitad del plan de estudios el conocimiento de la realidad que iba a conformar el ámbito profesional de los estudiantes. Entre los argumentos a favor de tal división se encontraba la falta de formación clínica de los estudiantes para aprovechar adecuadamente su presencia en el entorno sanitario [3]. Sin embargo, desde la primera experiencia publicada en 1976 [4], numerosos autores han desmentido esta asunción y es un hecho relativamente común que se facilite el contacto precoz con la realidad asistencial [5-7]. En Gran Bretaña, la implementación de programas de contacto precoz con la realidad asistencial ha sido en parte consecuencia de las recomendaciones que realizó el propio General Medical Council en 2009 [8].

En 2008 se inició el grado conjunto de medicina de la Universitat Autònoma de Barcelona con la Universitat Pompeu Fabra. Su plan de estudios se encontraba plenamente adaptado al Espacio Europeo de Educación Superior y aprovechó la oportunidad para introducir algunos cambios sustanciales respecto a los tradicionales. Entre ellos se encontraban unas asignaturas destinadas a permitir que los estudiantes conocieran antes del ciclo clínico las realidades de la asistencia primaria, las unidades de hospitalización con sus equipos de enfermería y los centros sociosanitarios y de salud mental $[9,10]$.

Un análisis preliminar de los resultados de esta experiencia con los estudiantes de primer curso se publicó en 2011 [11]. El objetivo de este artículo es presentar los resultados de los tres primeros cursos, una vez completada la experiencia, a fin de establecer la opinión de los estudiantes y la influencia del perfil profesional de los tutores en la adquisición de las competencias educativas.

\section{Sujetos y métodos}

\section{Entorno}

La experiencia se realizó en el marco del grado conjunto de medicina que ambas universidades iniciaron en el curso 2008-2009. Su plan de estudios contiene tres asignaturas, denominadas 'Prácticas de Grado I', 'Prácticas de Grado II' y 'Prácticas de Grado III', que se cursan, respectivamente en primer, segundo y tercer curso. En cada una, los estudiantes se dedican exclusivamente a una estancia de prácticas en un centro de asistencia primaria, en una unidad de hospitalización de enfermería y en un centro sociosanitario o de salud mental. El objetivo general de escoger tales entornos era diverso. En el caso de la asistencia primaria y la atención sociosanitaria y de salud mental, se deseaba que los estudiantes tuvieran un contacto precoz y directo con la atención extrahospitalaria. En el caso de la primera, la principal razón residía en que la asistencia primaria es el primer nivel de atención sanitaria y permite comprender una parte importante de la complejidad del sistema sanitario. En el segundo, se deseaba que los estudiantes comprendieran las peculiaridades de la atención de los enfermos crónicos y de edad avanzada, tanto los afectos de enfermedades más físicas como los que sufrían afecciones psiquiátricas. En el caso de las unidades de hospitalización, la razón era permitir que los alumnos contactasen con el nivel asistencial hospitalario, que constituye el otro gran pilar del sistema sanitario. Finalmente, de esta manera, se conseguía una visión global y completa de todo el sistema sanitario (hospitalización convencional, atención sociosanitaria, salud mental y atención primaria).

En cada curso, los estudiantes son asignados a uno de los ámbitos, de forma que hayan atendido las tres opciones al finalizar la mitad del grado. En cada período, los estudiantes se encuentran bajo la supervisión de un tutor o tutora, que generalmente es un médico o un profesional de enfermería, que se asigna aleatoriamente. Los detalles de las asignaturas, específicamente los requisitos académicos, los objetivos, las competencias que deben alcanzarse y el sistema de evaluación se describieron con detalle en Baños et al [11]. Brevemente, los estudiantes se incorporan de forma rotatoria y aleatoria a cada uno de los tres ámbitos. En cada caso acuden todas las tardes de 15:00 a 20:00 h durante un período de dos semanas, lo que supone un total de 50 horas. En estos centros se asigna un tutor por cada dos estudiantes que se encarga de su supervisión y asignación en las diferentes unidades funcionales 
de cada ámbito. Las actividades básicas, pero no exclusivas, de los estudiantes son la observación de la actividad asistencial y, a criterio de los profesionales, pueden realizar tareas de aprendizaje propedéutico. Una vez finalizada la estancia, los alumnos redactan un diario de prácticas y un portafolio que es evaluado por los profesores responsables de las asignaturas al finalizar cada periodo de estancia.

\section{Opinión de los estudiantes}

Una vez finalizadas las actividades docentes, en junio de 2012, se solicitó a los estudiantes de los tres primeros cursos que completaran voluntariamente una encuesta en que se les solicitaba su opinión sobre diversos aspectos de las asignaturas. En el primer grupo de preguntas se les solicitaba su grado de acuerdo mediante una escala de Likert de cinco opciones sobre los principales objetivos y competencias de las asignaturas, según figura en su plan de estudios (Tabla I). Se añadió un par de preguntas más para conocer su repercusión sobre la percepción de sus estudios ('después de estas prácticas considero que mi decisión de dedicarme a la medicina se ha reforzado' y 'considero que las prácticas de grado son una contribución importante a la formación de los estudiantes de medicina'). Luego se les pedía que puntuaran su grado de satisfacción (de 1 a 10) sobre diversos aspectos de organización de las asignaturas. Finalmente, los estudiantes de tercer curso, que habían completado las tres asignaturas, podían realizar una valoración de las prácticas de los tres años mediante escalas lineales visuales, con una puntuación de 0 a 10 (valoración global, contribución a su formación, conocimientos adquiridos).

\section{Análisis estadístico}

Los resultados de las encuestas se expresaron en forma de tablas descriptivas. Las respuestas cualitativas se han expresado con la frecuencia en números absolutos y porcentajes; el valor medio de cada variable, utilizando la mediana, dado que las respuestas cualitativas no seguían una distribución normal, y las respuestas cuantitativas (escalas lineales de 0-10), con media \pm desviación estándar.

Se hicieron dos análisis univariados, uno para establecer comparaciones entre las respuestas obtenidas en las encuestas y la profesión del tutor (enfermera o médico) y otro para establecer comparaciones entre los tres años evaluados. En estos análisis bivariados se han usado tablas de contingencia (test de $\chi^{2}$, con la corrección de Fisher si era necesaria) cuando las variables de las respuestas eran cua-
Tabla I. Objetivos educativos y competencias de las asignaturas de 'Prácticas de Grado' (entre paréntesis, la afirmación que la evaluaba en la encuesta de evaluación de la actividad).

Aplicar los valores personales y profesionales de excelencia, altruismo, sentido del deber, responsabilidad, integridad y honestidad en el ejercicio de la profesión ('las prácticas de grado me han permitido conocer los valores personales y profesionales de excelencia, altruismo, sentido del deber, responsabilidad y honestidad al ejercicio de la profesión')

Conocer los aspectos de la comunicación en pacientes, familiares y su entorno social ('las prácticas de grado me han permitido conocer las características principales de la comunicación con los pacientes, los familiares y su entorno social’')

Competencias Conocer los modelos de relación clínica -entrevista, comunicación verbal, comunicación no verbal-y sus interferencias ('las prácticas de grado me han permitido conocer los modelos de relación clínica y sus interferencias')

Conocer los fundamentos de la ética médica ('las prácticas de grado me han permitido conocer los fundamentos de la ética médica')

Comprender la importancia del trabajo en equipos multidisciplinares ('las prácticas de grado me han permitido comprender la importancia del trabajo en equipos multidisciplinares')

Tomar contacto con la realidad asistencial antes de la formación clínica ('las prácticas me han permitido conocer la realidad asistencial y el mundo de la actividad clínica')

Conocer los aspectos psicosociales vinculados a la salud ('después de estas prácticas comprendo mucho mejor los factores asociados a la enfermedad y las necesidades de los pacientes')

Objetivos

educativos Conocer las características del sistema sanitario y de los profesionales que intervienen ('las prácticas de grado me han permitido conocer algunas de las características de nuestro sistema sanitario y de los diferentes profesionales que intervienen')

Reflexionar sobre la complejidad de la relación entre pacientes y profesionales sanitarios ('las prácticas me han permitido conocer y reflexionar sobre la complejidad de la relación entre los pacientes y los profesionales sanitarios')

litativas, y las pruebas de Mann-Whitney y KruskalWallis (dado que las variables no seguían una distribución normal), cuando eran cuantitativas.

Finalmente, en el grupo de estudiantes que habían completado los tres cursos, se efectuó un análisis de las puntuaciones medias de las escalas lineales visuales $(0-10)$ correspondientes a cada curso. Para ello se efectuó una comparación de medias con datos independientes (aunque el alumno completaba tres veces la misma escala, ésta se correspondía con un curso y un destino diferente). En esta comparación de medias se utilizó una prueba de ANOVA y la prueba de Kruskal-Wallis.

\section{Resultados}

\section{Descripción de la muestra}

Participaron 124 estudiantes, $46(37,1 \%)$ de primer curso, $34(27,4 \%)$ de segundo y $44(35,5 \%)$ de terce- 
Tabla II. Opiniones de los estudiantes sobre competencias profesionales y objetivos educativos y otros aspectos asociados a las actividades, efectuados durante las prácticas ( $n=124$ ).

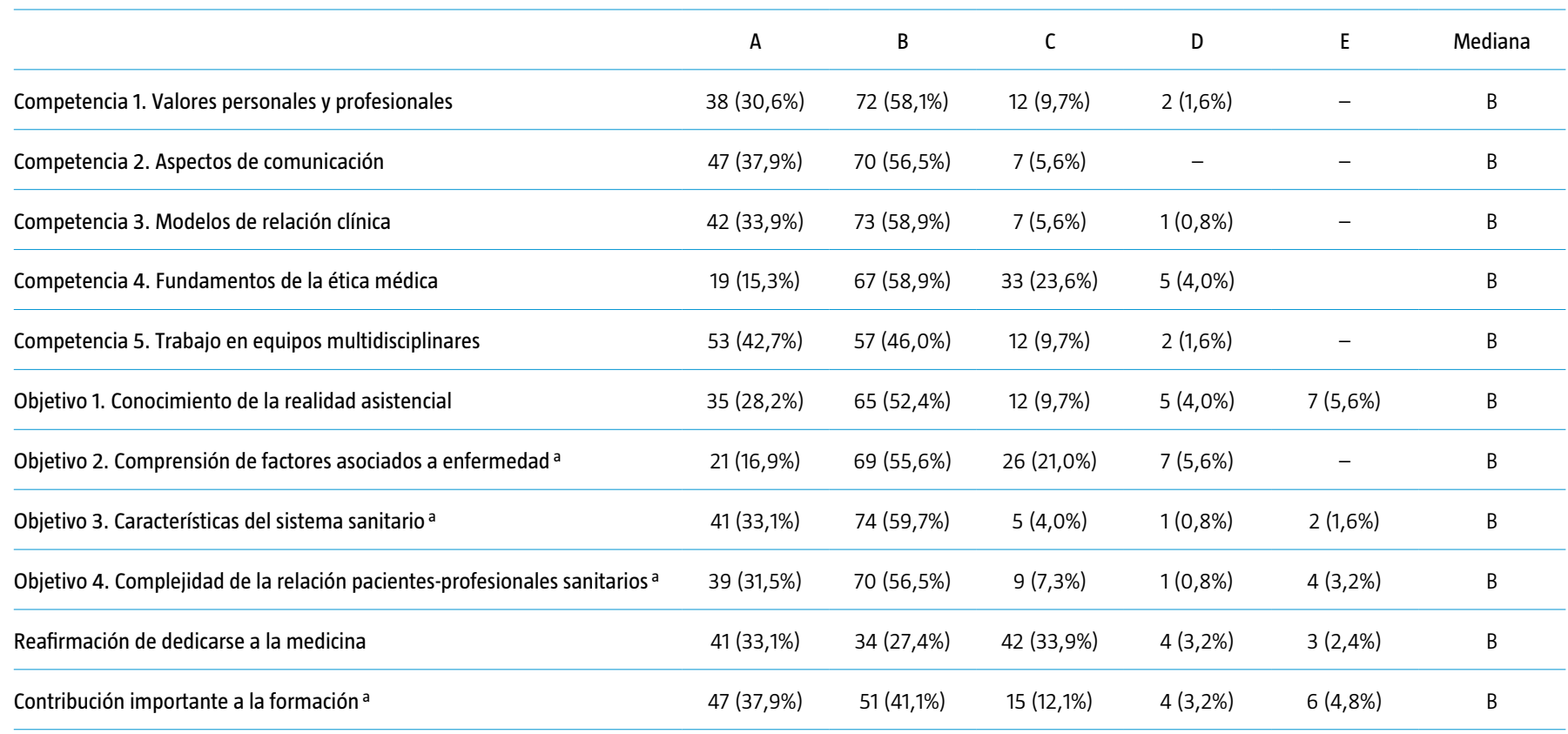

A: completamente de acuerdo; B: de acuerdo; C: ni de acuerdo ni en desacuerdo; D: en desacuerdo; E: completamente en desacuerdo. a Un caso inválido en cada fila.

ro. Sobre los estudiantes matriculados en los tres cursos $(n=187)$, supuso el $66,3 \%$, que se distribuyó de forma relativamente uniforme entre ellos (primero: $66,6 \%$; segundo: $57,6 \%$; tercero: $69 \%$ ).

El $67,7 \%$ eran mujeres. Su edad media fue de 21,1 \pm 3,92 años. En el curso anterior habían realizado las prácticas en un centro de asistencia primaria ( $n=40 ; 32,3 \%$ ), en un centro sociosanitario (CSS) o de asistencia mental $(n=39 ; 31,4 \%$. CSS Fòrum: $n=37 ; 29,8 \%$. CSS Centre Assistencial Dr. Emili Mira i López: $n=2 ; 1,6 \%$ ) o en un equipo de enfermería de una planta de hospitalización $(n=45$; $36,2 \%$. Hospital del Mar: $n=23 ; 18,5 \%$. Hospital de l'Esperança: $n=22 ; 17,7 \%)$. El 52,4\% $(n=65)$ fueron tutorizados por médicos, y el $47,6 \%(n=59)$, por personal de enfermería.

\section{Opiniones de la muestra global de estudiantes}

La tabla II recoge las respuestas de toda la muestra de estudiantes respecto a las competencias profesionales y otros objetivos educativos, destacando que la mediana para todas las afirmaciones fue 'de acuerdo.' Cuando se agruparon las respuestas 'completamente de acuerdo' y 'de acuerdo', en todos los casos se superó el $60 \%$. En tres casos, esta agrupación superó el $90 \%$ en las referidas a 'características de comunicación con pacientes y familiares' $(94,4 \%)$ (competencia 2), 'modelos de relación clínica' (92,8\%) (competencia 3) y 'conocimiento del sistema sanitario y sus profesionales' (92,8\%) (objetivo 3). Las que obtuvieron valores más bajos fueron 'reafirmación en dedicarme a la medicina' (60,5\%) y 'comprensión de factores asociados a enfermedad' (72,5\%) (objetivo 2).

En la tabla III se recogen los resultados separados por cursos. Sólo se observaron diferencias en dos afirmaciones: 'conocer los modelos de relación clínica' $(p<0,05)$ y 'las prácticas de grado son una contribución importante a la formación de los estudiantes de medicina' $(p<0,01)$. En ambos casos, los estudiantes de tercer curso puntuaron por debajo de los de primero y segundo. En el caso de la segunda afirmación, también se observaron diferencias entre primero y segundo, con valores inferiores en estos últimos.

La tabla IV resume los valores obtenidos en la evaluación de diversos aspectos relacionados con las prácticas. Excepto en la variable 'horario', el resto superó el valor de 7 , con un máximo en 'atención de tutores' $(8,28 \pm 1,61)$ y 'relación con los pacientes' $(7,93 \pm 1,46)$. Cuando los resultados se separaron por cursos, se mantuvieron los valores superiores a 7 para todas las variables (excepto 'horario') en 
Tabla III. Opiniones de los estudiantes sobre las competencias profesionales y los objetivos educativos y otros aspectos asociados a las actividades llevados a cabo durante las prácticas, según el curso de cada estudiante $(n=124)$.

\begin{tabular}{|c|c|c|c|c|c|c|c|}
\hline & & A & B & c & D & E & $p$ \\
\hline \multirow{3}{*}{$\begin{array}{l}\text { Competencia } 1 . \\
\text { Valores personales } \\
\text { y profesionales }\end{array}$} & 1er. curso & 15 (32,6\%) & $28(60,9 \%)$ & $2(4,3 \%)$ & $1(2,2 \%)$ & - & \multirow{3}{*}{0,65} \\
\hline & $2 .{ }^{\circ}$ curso & $12(35,3 \%)$ & $18(52,9 \%)$ & $4(11,8 \%)$ & - & - & \\
\hline & 3er. curso & $11(25,0 \%)$ & $26(59,1 \%)$ & $6(13,6 \%)$ & $1(2,2 \%)$ & - & \\
\hline \multirow{3}{*}{$\begin{array}{l}\text { Competencia } 2 . \\
\text { Aspectos de comunicación }\end{array}$} & 1er. curso & $22(47,8 \%)$ & $22(47,8 \%)$ & $2(4,3 \%)$ & - & - & \multirow{3}{*}{0,06} \\
\hline & $2 .{ }^{\circ}$ curso & $14(41,2 \%)$ & $20(58,8 \%)$ & - & - & - & \\
\hline & 3er. curso & $11(25,0 \%)$ & $28(63,6 \%)$ & $5(11,4 \%)$ & - & - & \\
\hline \multirow{3}{*}{$\begin{array}{l}\text { Competencia } 3 \text {. } \\
\text { Modelos de relación clínica }\end{array}$} & 1er. curso & $16(34,8 \%)$ & $29(63,0 \%)$ & $1(2,2 \%)$ & - & - & \multirow{3}{*}{$<0,05$} \\
\hline & 2. .9 curso & $16(47,1 \%)$ & $18(52,9 \%)$ & - & - & - & \\
\hline & 3er. curso & $10(22,7 \%)$ & $26(59,1 \%)$ & $6(13,6 \%)$ & $1(2,3 \%)$ & $1(2,3 \%)$ & \\
\hline \multirow{3}{*}{$\begin{array}{l}\text { Competencia } 4 . \\
\text { Fundamentos de la ética médica }\end{array}$} & 1er. curso & $9(19,6 \%)$ & $23(50,0 \%)$ & $13(28,3 \%)$ & $1(2,2 \%)$ & - & \multirow{3}{*}{0,20} \\
\hline & $2 .{ }^{\circ}$ curso & $5(14,7 \%)$ & $23(67,6 \%)$ & $4(11,8 \%)$ & $2(5,9 \%)$ & - & \\
\hline & 3er. curso & $5(11,4 \%)$ & $21(47,7 \%)$ & $16(36,4 \%)$ & $2(4,5 \%)$ & - & \\
\hline \multirow{3}{*}{$\begin{array}{l}\text { Competencia } 5 . \\
\text { Trabajo en equipos } \\
\text { multidisciplinares }\end{array}$} & 1er. curso & 19 (41,3\%) & $23(50,0 \%)$ & $3(6,5 \%)$ & $1(2,2 \%)$ & - & \multirow{3}{*}{0,64} \\
\hline & $2 .^{\circ}$ curso & $15(44,1 \%)$ & $13(38,2 \%)$ & $6(17,6 \%)$ & - & - & \\
\hline & 3er. curso & $19(43,2 \%)$ & $21(47,7 \%)$ & $3(6,8 \%)$ & $1(2,3 \%)$ & - & \\
\hline \multirow{3}{*}{$\begin{array}{l}\text { Objetivo } 1 . \\
\text { Conocimiento de } \\
\text { la realidad asistencial }\end{array}$} & 1er. curso & $11(23,9 \%)$ & $26(56,5 \%)$ & $5(10,9 \%)$ & $2(4,3 \%)$ & $2(4,3 \%)$ & \multirow{3}{*}{0,51} \\
\hline & $2 .{ }^{\circ}$ curso & $6(17,6 \%)$ & $21(61,8 \%)$ & $4(11,8 \%)$ & $1(2,9 \%)$ & $2(5,9 \%)$ & \\
\hline & 3er. curso & $18(40,9 \%)$ & $18(40,9 \%)$ & $3(6,8 \%)$ & $2(4,5 \%)$ & $3(6,8 \%)$ & \\
\hline \multirow{3}{*}{$\begin{array}{l}\text { Objetivo } 2 \text {. } \\
\text { Comprensión de factores } \\
\text { asociados a enfermedad }{ }^{\text {a }}\end{array}$} & 1er. curso & $6(13,0 \%)$ & $26(56,5 \%)$ & $11(23,9 \%)$ & $3(6,5 \%)$ & - & \multirow{3}{*}{0,47} \\
\hline & $2 .{ }^{\circ}$ curso & $4(12,1 \%)$ & $23(69,7 \%)$ & $5(15,2 \%)$ & $1(3,0 \%)$ & - & \\
\hline & 3er. curso & $11(25,0 \%)$ & $20(45,5 \%)$ & $10(22,7 \%)$ & $3(8,6 \%)$ & - & \\
\hline \multirow{3}{*}{$\begin{array}{l}\text { Objetivo } 3 \text {. } \\
\text { Características del } \\
\text { sistema sanitario }^{\text {a }}\end{array}$} & 1er. curso & $16(34,8 \%)$ & $26(56,5 \%)$ & $3(6,5 \%)$ & $1(2,2 \%)$ & - & \multirow{3}{*}{0,30} \\
\hline & $2 .^{\circ}$ curso & $9(27,3 \%)$ & $21(63,6 \%)$ & $2(6,1 \%)$ & - & $1(3,0 \%)$ & \\
\hline & 3er. curso & $16(36,4 \%)$ & $27(61,4 \%)$ & - & - & $1(2,3 \%)$ & \\
\hline \multirow{3}{*}{$\begin{array}{l}\text { Objetivo } 4 . \\
\text { Complejidad de la relación } \\
\text { paciente-profesional sanitario a }\end{array}$} & 1er. curso & $13(28,3 \%)$ & $26(56,5 \%)$ & $6(13,0 \%)$ & - & $1(2,2 \%)$ & \multirow{3}{*}{0,68} \\
\hline & $2 .{ }^{\circ}$ curso & $11(33,3 \%)$ & $19(57,6 \%)$ & $2(6,1 \%)$ & - & $1(3,0 \%)$ & \\
\hline & 3er. curso & $15(34,1 \%)$ & $25(56,8 \%)$ & $1(2,3 \%)$ & $1(2,3 \%)$ & $2(4,5 \%)$ & \\
\hline \multirow{3}{*}{$\begin{array}{l}\text { Reafirmación de } \\
\text { dedicarse a la medicina }\end{array}$} & 1er. curso & $18(39,1 \%)$ & $10(21,7 \%)$ & $17(37,0 \%)$ & $1(2,2 \%)$ & - & \multirow{3}{*}{0,70} \\
\hline & $2 .{ }^{\circ}$ curso & $9(28,5 \%)$ & $12(35,3 \%)$ & $10(29,4 \%)$ & $1(2,9 \%)$ & $2(5,9 \%)$ & \\
\hline & 3er. curso & $14(31,8 \%)$ & $12(27,3 \%)$ & $15(34,1 \%)$ & $2(4,5 \%)$ & $1(2,3 \%)$ & \\
\hline \multirow{3}{*}{$\begin{array}{l}\text { Contribución } \\
\text { importante a la formación a }\end{array}$} & 1er. curso & $30(65,2 \%)$ & $13(28,3 \%)$ & $1(2,2 \%)$ & - & $2(4,3 \%)$ & \multirow{3}{*}{$<0,01$} \\
\hline & $2 .{ }^{\circ}$ curso & $11(33,3 \%)$ & $15(45,5 \%)$ & $5(15,2 \%)$ & - & $2(6,1 \%)$ & \\
\hline & 3er. curso & $6(13,6 \%)$ & $23(52,3 \%)$ & $9(20,5 \%)$ & $4(9,1 \%)$ & $2(4,5 \%)$ & \\
\hline
\end{tabular}

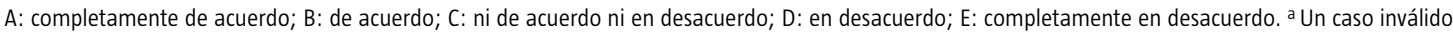
en cada fila. 
Tabla IV. Grado de satisfacción (0: ninguna; 10: completa) en diversos aspectos relacionados con las prácticas evaluados mediante una escala numérica, con valores expresados como media \pm desviación estándar. Los valores de $p$ indican las diferencias entre primer, segundo y tercer curso $(n=124)$.

\begin{tabular}{lccccc}
\hline & $\begin{array}{c}\text { Puntuación } \\
\text { global }\end{array}$ & $\begin{array}{c}\text { Primer } \\
\text { curso }\end{array}$ & $\begin{array}{c}\text { Segundo } \\
\text { curso }\end{array}$ & $\begin{array}{c}\text { Tercer } \\
\text { curso }\end{array}$ & $p$ \\
\hline Organización & $7,03 \pm 1,65$ & $7,13 \pm 1,54$ & $7,15 \pm 1,78$ & $6,84 \pm 1,68$ & 0,37 \\
\hline Horario & $6,25 \pm 2,01$ & $6,46 \pm 1,96$ & $6,52 \pm 2,15$ & $5,84 \pm 1,95$ & 0,08 \\
\hline Atención de los tutores & $8,28 \pm 1,61$ & $8,63 \pm 1,56$ & $8,69 \pm 1,59$ & $7,61 \pm 1,48$ & $<0,01$ \\
\hline $\begin{array}{l}\text { Relación con los pacientes } \\
\text { Aprendizaje }\end{array}$ & $7,93 \pm 1,46$ & $8,28 \pm 1,37$ & $8,12 \pm 1,29$ & $7,41 \pm 1,56$ & 0,02 \\
\hline $\begin{array}{l}\text { Experiencia vital } \\
\text { Comprensión de la }\end{array}$ & $7,35 \pm 1,70$ & $7,74 \pm 1,81$ & $7,91 \pm 1,18$ & $6,52 \pm 1,62$ & $<0,01$ \\
\hline $\begin{array}{l}\text { Conocimiento de los } \\
\text { procesos asistenciales }\end{array}$ & $7,72 \pm 1,62$ & $8,30 \pm 1,38$ & $8,03 \pm 1,15$ & $6,89 \pm 1,82$ & $<0,01$ \\
\hline $\begin{array}{l}\text { Comprensión de las otras } \\
\text { profesiones sanitarias }\end{array}$ & $7,64 \pm 1,61$ & $7,61 \pm 1,94$ & $7,76 \pm 1,17$ & $7,59 \pm 1,54$ & 0,90 \\
\hline $\begin{array}{l}\text { Conocimiento de los } \\
\text { centros asistenciales }\end{array}$ & $7,72 \pm 1,08$ & $7,54 \pm 1,20$ & $7,76 \pm 0,79$ & $7,89 \pm 1,14$ & 0,36 \\
\hline & $7,41 \pm 0,69$ & $7,64 \pm 0,69$ & $7,61 \pm 1,31$ & 0,46 \\
\hline
\end{tabular}

los estudiantes de primer y segundo curso. En tercero, sin embargo, tampoco alcanzaron este valor las variables 'aprendizaje,', 'organización' y 'experiencia vital', aunque todas superaron el valor de 6,5. Al comparar los valores por cursos, se observaron puntuaciones significativamente inferiores en alumnos de tercer curso respecto a los otros dos en las variables 'atención de tutores' $(p<0,01)$, 'relación con pacientes' $(p<0,05)$, 'aprendizaje' $(p<0,01) \mathrm{y}$ 'experiencia vital' $(p<0,01)$.

\section{Opiniones de los estudiantes según el tutor}

La tabla V muestra el análisis comparativo de los resultados respecto a las competencias profesionales y otros objetivos educativos según que el tutor fuera un médico o un profesional de enfermería. Destaca que no existieron diferencias en ninguna de las afirmaciones planteadas, excepto la referida a 'conocimiento y reflexión sobre la complejidad de las relaciones entre pacientes y profesionales sanitarios' $(p<0,05)$, donde los porcentajes agrupados de las respuestas 'completamente de acuerdo' y 'de acuerdo' fueron del 94,9\% y $82,9 \%$ para profesionales de enfermería y médicos, respectivamente. En la tabla VI se recoge el grado de satisfacción en función de la profesión del tutor/a. No se apreciaron diferencias excepto en la variable 'comprensión de otras profesiones sanitarias' $(p<0,01)$, con valores de $8,24 \pm 1,05$ y $7,09 \pm 1,84$ para profesionales de enfermería y médicos, respectivamente.

\section{Opiniones de los estudiantes que habían completado los tres cursos}

La tabla VII recoge la valoración comparativa de los tres años. En la valoración global, la máxima puntuación se alcanza en los centros de asistencia primaria y los CSS, mientras que las estancias en unidades hospitalarias obtienen un valor significativamente inferior. En la variable 'contribución de las prácticas a la formación', los centros de asistencia primaria ocupan el primer lugar, con diferencias significativas respecto a los centros hospitalarios. También se observaron diferencias estadísticas entre los CSS y de salud mental con los centros hospitalarios. En la tercera variable, 'valoración de los conocimientos adquiridos', de nuevo los centros de asistencia primaria obtienen valores significativamente superiores al compararlos con el resto. Se observaron diferencias significativas entre centros de asistencia primaria y CSS/salud mental con los hospitalarios, pero no entre los dos primeros.

\section{Discusión}

Los resultados positivos expuestos en el presente artículo confirman y extienden los publicados previamente en un estudio piloto realizado sólo con estudiantes de primer curso [11]. Además, revelan aspectos interesantes, como el hecho de que no hubo diferencias en la consecución de las competencias y los objetivos de las asignaturas en función de la profesión del tutor (médico o personal de enfermería). Así mismo, el presente estudio aporta diferencias en algunos aspectos entre los estudiantes de distintos cursos, como la presencia de un mayor nivel de satisfacción de las prácticas realizadas en el ámbito de la asistencia primaria.

Los resultados de la encuesta global (Tabla II) sirven para reafirmar que la iniciativa de proporcionar a los estudiantes un espacio de contacto precoz con el sistema sanitario, en los primeros cursos, tiene gran aceptación, con valoraciones muy positivas. Adicionalmente, estos hallazgos permiten consolidar las tres asignaturas como un paso importante para que los estudiantes vean la transición entre el periodo preclínico y el clínico de una manera más 
Tabla V. Opiniones de los estudiantes sobre las competencias profesionales y los objetivos educativos y otros aspectos asociados a las actividades llevados a cabo durante las prácticas, según la profesión del tutor $(n=124)$. Los valores de $p$ indican las diferencias entre la profesión del tutor.

\begin{tabular}{|c|c|c|c|c|c|c|c|}
\hline & Profesión del tutor & $A$ & B & C & D & $\mathrm{E}$ & $p$ \\
\hline \multirow{2}{*}{$\begin{array}{l}\text { Competencia } 1 . \\
\text { Valores personales y profesionales }\end{array}$} & Enfermera/o & $20(33,9 \%)$ & $30(50,8 \%)$ & $7(11,9 \%)$ & $2(3,4 \%)$ & - & \multirow{2}{*}{0,26} \\
\hline & Médico/a & $18(27,7 \%)$ & $42(64,6 \%)$ & $5(7,7 \%)$ & - & - & \\
\hline \multirow{2}{*}{$\begin{array}{l}\text { Competencia } 2 . \\
\text { Aspectos de comunicación }\end{array}$} & Enfermera/o & $18(30,5 \%)$ & $37(62,7 \%)$ & $4(6,8 \%)$ & - & - & \multirow{2}{*}{0,26} \\
\hline & Médico/a & $29(44,6 \%)$ & $33(50,8 \%)$ & $3(4,6 \%)$ & - & - & \\
\hline \multirow{2}{*}{$\begin{array}{l}\text { Competencia } 3 . \\
\text { Modelos de relación clínica }\end{array}$} & Enfermera/o & $17(28,8 \%)$ & $37(62,7 \%)$ & $3(5,1 \%)$ & $1(1,7 \%)$ & $1(1,7 \%)$ & \multirow{2}{*}{0,51} \\
\hline & Médico/a & $25(38,5 \%)$ & $36(55,4 \%)$ & $4(6,2 \%)$ & - & - & \\
\hline \multirow{2}{*}{$\begin{array}{l}\text { Competencia } 4 . \\
\text { Fundamentos de la ética médica }\end{array}$} & Enfermera/o & $10(16,9 \%)$ & $29(49,2 \%)$ & $17(28,8 \%)$ & $3(5,1 \%)$ & - & \multirow{2}{*}{0,78} \\
\hline & Médico/a & $9(13,8 \%)$ & $38(58,5 \%)$ & $16(24,6 \%)$ & $2(83,1 \%)$ & & \\
\hline \multirow{2}{*}{$\begin{array}{l}\text { Competencia } 5 . \\
\text { Trabajo en equipos } \\
\text { multidisciplinares }\end{array}$} & Enfermera/o & $31(52,4 \%)$ & $22(37,3 \%)$ & $6(10,2 \%)$ & - & - & \multirow{2}{*}{0,09} \\
\hline & Médico/a & $22(33,8 \%)$ & $35(53,8 \%)$ & $6(9,2 \%)$ & $2(3,1 \%)$ & - & \\
\hline \multirow{2}{*}{$\begin{array}{l}\text { Objetivo } 1 . \\
\text { Conocimiento de } \\
\text { la realidad asistencial }\end{array}$} & Enfermera/o & $17(28,8 \%)$ & 31 (52,5\%) & 7 (11,9\%) & $2(3,4 \%)$ & $2(3,4 \%)$ & \multirow{2}{*}{0,82} \\
\hline & Médico/a & $18(27,7 \%)$ & $34(52,3 \%)$ & $5(7,7 \%)$ & $3(4,6 \%)$ & $5(7,7 \%)$ & \\
\hline \multirow{2}{*}{$\begin{array}{l}\text { Objetivo } 2 \text {. } \\
\text { Comprensión de factores } \\
\text { asociados a enfermedad a }\end{array}$} & Enfermera/o & $13(22,0 \%)$ & $34(57,6 \%)$ & $10(16,9 \%)$ & $2(3,4 \%)$ & - & \multirow{2}{*}{0,32} \\
\hline & Médico/a & $8(12,5 \%)$ & $35(54,7 \%)$ & $16(25,0 \%)$ & $5(7,8 \%)$ & - & \\
\hline \multirow{2}{*}{$\begin{array}{l}\text { Objetivo } 3 . \\
\text { Características del } \\
\text { sistema sanitario }{ }^{\text {a }}\end{array}$} & Enfermera/o & $18(30,5 \%)$ & $37(62,7 \%)$ & $4(6,8 \%)$ & - & - & \multirow{2}{*}{0,28} \\
\hline & Médico/a & $23(35,9 \%)$ & $37(57,8 \%)$ & $1(1,6 \%)$ & $1(1,6 \%)$ & $2(3,1 \%)$ & \\
\hline \multirow{2}{*}{$\begin{array}{l}\text { Objetivo } 4 . \\
\text { Complejidad de la relación } \\
\text { paciente-profesional sanitario a }\end{array}$} & Enfermera/o & $14(23,7 \%)$ & $42(71,2 \%)$ & $2(3,4 \%)$ & - & $1(1,7 \%)$ & \multirow{2}{*}{$<0,05$} \\
\hline & Médico/a & $25(39,1 \%)$ & $28(43,8 \%)$ & $7(10,9 \%)$ & $1(1,6 \%)$ & $3(4,7 \%)$ & \\
\hline \multirow{2}{*}{$\begin{array}{l}\text { Reafirmación de } \\
\text { dedicarse a la medicina }\end{array}$} & Enfermera/o & $22(37,3 \%)$ & $14(23,7 \%)$ & $21(35,6 \%)$ & $1(1,7 \%)$ & $1(1,7 \%)$ & \multirow{2}{*}{0,69} \\
\hline & Médico/a & $19(29,2 \%)$ & $20(30,8 \%)$ & $21(21,3 \%)$ & $3(4,6 \%)$ & $2(3,1 \%)$ & \\
\hline \multirow{2}{*}{$\begin{array}{l}\text { Contribución } \\
\text { importante a la formación a }\end{array}$} & Enfermera/o & $21(35,6 \%)$ & $26(44,1 \%)$ & $9(15,3 \%)$ & $2(3,4 \%)$ & $1(1,7 \%)$ & \multirow{2}{*}{0,47} \\
\hline & Médico/a & $26(40,6 \%)$ & 25 (39,1\%) & $6(9,4 \%)$ & $2(3,1 \%)$ & $5(7,8 \%)$ & \\
\hline
\end{tabular}

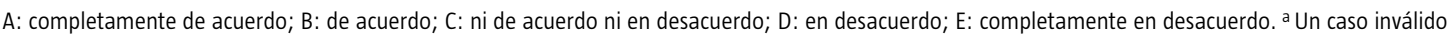
en cada fila.

integrada, comprendiendo mejor cómo algunos conocimientos básicos pueden aplicarse a la realidad asistencial. Estos resultados coinciden con los observados por otros autores que aplicaron experiencias similares $[3,12,13]$.

Por otro lado, es importante resaltar el impacto positivo que tiene sobre los estudiantes el hecho de contactar con la realidad profesional de forma tan precoz. Aunque en el presente estudio no se han evaluado específicamente aspectos emocionales, muchas de las respuestas del cuestionario reflejan una percepción positiva de su futura actividad profesional. Por ejemplo, en la tabla II, el $88,7 \%$ de los estudiantes están 'completamente de acuerdo' y 'de acuerdo' en que las prácticas les han permitido acercarse a los valores personales y profesionales del 
Tabla VI. Grado de satisfacción (0: ninguna; 10: completa) en diversos aspectos asociados con las prácticas evaluados mediante una escala numérica, expresada como media \pm desviación estándar, según la profesión del tutor $(n=124)$.

\begin{tabular}{lccc}
\hline & Enfermería & Medicina & $p$ \\
\hline Organización & $6,90 \pm 1,87$ & $7,16 \pm 1,42$ & 0,62 \\
\hline Horario & $5,92 \pm 2,21$ & $6,56 \pm 1,78$ & 0,17 \\
\hline Atención de los tutores & $8,46 \pm 1,79$ & $8,11 \pm 1,41$ & 0,05 \\
\hline Relación con los pacientes & $8,08 \pm 1,45$ & $7,68 \pm 1,47$ & 0,23 \\
\hline Aprendizaje & $7,25 \pm 1,63$ & $7,44 \pm 1,77$ & 0,37 \\
\hline Experiencia vital & $7,63 \pm 1,56$ & $7,72 \pm 1,68$ & 0,93 \\
\hline Comprensión de la profesión médica & $6,76 \pm 2,22$ & $7,68 \pm 1,33$ & 0,13 \\
\hline Comprensión de las otras profesiones sanitarias & $8,24 \pm 1,05$ & $7,09 \pm 1,84$ & $<0,01$ \\
\hline Conocimiento de los centros asistenciales & $7,88 \pm 1,11$ & $7,58 \pm 1,05$ & 0,07 \\
\hline Conocimiento de los procesos asistenciales & $7,61 \pm 1,13$ & $7,48 \pm 1,19$ & 0,44 \\
\hline
\end{tabular}

ejercicio de la profesión (competencia 1; tabla I). El $60 \%$ de los estudiantes también están 'completamente de acuerdo' y 'de acuerdo' en que estas prácticas les han servido para reafirmar su vocación (pregunta sobre la reafirmación de dedicarse a la medicina). Por otra parte, los responsables de las asignaturas y también muchos de los profesionales que han ejercido de tutores han podido comprobar, en la lectura de los diarios de prácticas y de los portafolios que los alumnos escriben al finalizar la asignatura, el impacto positivo que las prácticas han ejercido sobre las actitudes de los estudiantes. Aunque el análisis de estos ejercicios no ha sido objeto del presente trabajo, los autores desean hacer constar que dichos escritos contenían numerosas reflexiones positivas a favor del ejercicio de las profesiones sanitarias (médica y enfermería), así como otros razonamientos y reflexiones sobre historias de pacientes, sus familias y el funcionamiento del sistema sanitario. Aunque las 'emociones positivas' relacionadas con el ejercicio de la profesión no forman parte estricta del currículo de conocimientos y capacidades que han de aportarse a los estudiantes, nadie puede negar el potente efecto positivo que ejerce sobre el aprendizaje la existencia de una buena motivación, más aún en una profesión tan vocacional como debe ser la medicina.

Al comparar los resultados de las encuestas de los tres cursos se observa que, aunque en todas las preguntas la mayoría de los estudiantes están 'completamente de acuerdo' y 'de acuerdo' (Tabla III), parece existir una tendencia en los estudiantes de tercer curso a responder en algunas preguntas las opciones 'de acuerdo' y 'ni de acuerdo ni en desacuerdo'. Esta tendencia sólo alcanza significación estadística en dos preguntas: una, relacionada con el conocimiento de los modelos de relación clínica, y la otra, con la percepción de que las prácticas son una contribución importante para la formación. La explicación de esta tendencia podría relacionarse con el hecho de que los estudiantes, al llegar al tercer curso, ya han cursado las asignaturas de primero y segundo y ya han tenido dos experiencias previas de contacto precoz con el sistema sanitario; por ello, estos estudiantes partirían de una situación previa de mayor conocimiento del sistema, siendo entonces posible que la experiencia del tercer curso ejerciera, en algunos aspectos, un menor impacto en su formación. En este mismo sentido apuntan los resultados que se muestran en la tabla IV, en donde las puntuaciones correspondientes a las dimensiones de aprendizaje y experiencia vital son significativamente inferiores en este grupo. En cualquier caso, y como apuntan Smithson et al [13], es muy importante la planificación de actividades y los aspectos de organización, lo que implica reconocer que aquello que precisarían los estudiantes de tercer curso podría ser distinto de lo necesitado por estudiantes de los cursos previos.

Como se muestra en la tabla I, las competencias y objetivos educativos a conseguir en estas asignaturas son de carácter general y fundamentadas en el conocimiento del sistema sanitario, su funcionamiento y los profesionales que lo componen. En este sentido, los tutores de los alumnos obviamente debían ser profesionales sanitarios, pero no obligatoriamente médicos. Por ello, los estudiantes fueron tutorizados indistintamente por médicos o personal de enfermería según la unidad o centro asignado. Este hecho no ha supuesto diferencias para los estudiantes, ya que la gran mayoría de las respuestas de la encuesta han sido similares por los que han sido tutorizados por unos o por otros (Tabla V). Únicamente hubo diferencias significativas en la pregunta correspondiente a la comprensión de la complejidad en la relación entre pacientes y profesionales sanitarios, en la que hubo un mayor porcentaje de alumnos que parecían estar mas 'de acuerdo' en haber cumplido este objetivo y que habían sido tutorizados por personal de enfermería. Probablemente ello pueda deberse al hecho de que este personal permanece mucho más tiempo en contacto con los pacientes, lo que favorece que puedan transmitir con ma- 
yor facilidad las sutilezas de tal relación. Por otra parte, y como es previsible, estos alumnos afirmaron más frecuentemente haber comprendido mejor otras profesiones sanitarias que los que habían estado con médicos (Tabla VI). Aunque desconocemos la existencia de estudios publicados en los que los profesionales de enfermería hayan actuado como tutores de estudiantes de medicina, algunos autores han citado el interés de que los médicos más jóvenes participen en las actividades docentes de contacto precoz [14]. Ello sugeriría que el amplio conocimiento conceptual no sería un determinante en la selección de tutores en este momento de formación de los estudiantes, y que éstos podrían beneficiarse de la interacción con otro perfil de profesionales.

Los estudiantes de tercer curso que ya habían completado los tres ciclos de prácticas otorgaron puntuaciones significativamente inferiores a las prácticas realizadas en el ámbito del hospital (Tabla VII). Esto pudo deberse a varios factores; en primer lugar, las prácticas se realizaban únicamente por las tardes, hecho que podría haber justificado una menor percepción en el aprendizaje porque muchas de las actividades propias de la atención hospitalaria se realizan por la mañana y la actividad de las tardes puede resultar más rutinaria y menos atractiva. Esto no era así en los centros de atención primaria y CSS, en donde no había tanta diferencia entre actividades de mañana y tarde. En segundo lugar, es posible que los entornos de la atención primaria, sociosanitario y salud mental, por ser más desconocidos para los estudiantes, les pudiesen resultar más novedosos y atractivos, al menos en esta fase tan precoz de su formación. Por último, hubo algunos grupos de estudiantes cuya estancia coincidió con periodos de cierre de camas y traslados de unidades, hechos que podrían haber influido en que el alumno tuviese una percepción menos positiva de su aprendizaje.

Como posibles limitaciones al presente estudio, se exponen a continuación una serie de comentarios y reflexiones. De los alumnos que respondieron a las encuestas, poco más de un tercio había realizado los tres cursos. Este hecho puede haber influido en la valoración global de las encuestas, ya que es posible que la opinión del alumno y la percepción subjetiva de su aprendizaje esté influida por su propia experiencia acumulada. Dicho de otra manera, el contacto precoz que proporcionan las prácticas a un alumno de primero no puede considerarse 'igual de precoz' cuando el alumno está en tercero. Así mismo, pueden existir otros factores que influyan de manera distinta en los diferentes grupos de estudiantes (orden de rotación, circunstan-
Tabla VII. Evaluación comparativa (0: muy mala; 10: muy buena) de los alumnos de tercer curso que han finalizado los tres períodos de prácticas $(n=44)$, expresado como media \pm desviación estándar.

\begin{tabular}{lccc}
\hline & $\begin{array}{c}\text { Centros } \\
\text { de primaria }\end{array}$ & $\begin{array}{c}\text { Centros sociosanitarios } \\
\text { y de salud mental }\end{array}$ & $\begin{array}{c}\text { Hospitales (plantas } \\
\text { de hospitalización) }\end{array}$ \\
\hline Valoración global & $7,84 \pm 1,18^{\mathrm{a}}$ & $7,93 \pm 1,08^{\mathrm{a}}$ & $6,48 \pm 2,09$ \\
\hline $\begin{array}{l}\text { Contribución de las } \\
\text { prácticas a la formación }\end{array}$ & $7,59 \pm 1,30^{\mathrm{a}}$ & $7,15 \pm 1,62^{\mathrm{a}}$ & $5,93 \pm 2,17$ \\
\hline $\begin{array}{l}\text { Valoración de los } \\
\text { conocimientos adquiridos }\end{array}$ & $7,70 \pm 1,47^{\mathrm{b}}$ & $7,20 \pm 1,49^{\mathrm{c}}$ & $6,31 \pm 2,31$ \\
\hline
\end{tabular}

a Centros de primaria frente a hospitales, centros sociosanitarios y de salud mental frente a hospitales $(p<0,001)$; ${ }^{b}$ Centros de primaria frente a hospitales $(p<0,01)$; ' Centros sociosanitarios y de salud mental frente a hospitales $(p<0,05)$

cias concretas del entorno, preferencias personales del estudiante, afinidades y actividades del tutor asignado). Por todo ello, para minimizar la influencia de todos estos factores y poder establecer comparaciones entre diferentes cursos, en el futuro sería necesario disponer de un mayor número de alumnos encuestados de tercer curso con un mismo nivel de experiencia acumulada, para que puedan opinar sobre los tres entornos de prácticas en mayor igualdad de condiciones.

En relación a la tutorización de estudiantes de medicina por profesionales de enfermería, cabe mencionar que ha sido una experiencia positiva y novedosa en nuestro entorno, aunque en su inicio suscitó cierta polémica. Como se ha argumentado, las competencias a adquirir en estas asignaturas no son específicas ni exclusivas de la profesión médica, sino genéricas y transversales, propias de todas las profesiones sanitarias. Tampoco los objetivos de la asignatura incluyen conocimientos propios y exclusivos del médico, sino conocimientos generales que competen a todos los profesionales de la salud (por ejemplo, contacto con la realidad asistencial, aspectos psicosociales que rodean al paciente, características del sistema sanitario). Todos los tutores dispusieron de un listado de actividades recomendadas con la intención de que las prácticas se centrasen en las competencias deseadas, evitando entrar en otras competencias más específicas que no eran propias de estas asignaturas [11]. Por ello, los estudiantes tutorizados por personal de enfermería no mostraron diferencias significativas en la gran mayoría de las respuestas de la encuesta al ser comparados con los que habían estado con médicos.

Los resultados del trabajo permiten afirmar que, después de los tres primeros años de experiencia en 
el contacto precoz con la realidad asistencial en estudiantes de medicina, las opiniones de los profesores responsables, tutores y estudiantes coinciden en que la experiencia tiene muchos aspectos positivos desde el punto de vista de la adquisición de conocimientos sobre el sistema sanitario y de las profesiones que lo componen, y desde el punto de vista motivacional y afectivo $[15,16]$. Queda por ver si en el futuro esta experiencia supone una ventaja real al llegar al periodo clínico de su aprendizaje, o bien si los conocimientos adquiridos en esta fase de contacto precoz permiten al alumno despertar intereses profesionales que de otra manera difícilmente se hubiesen planteado. La complejidad del sistema sanitario actual, con sus diferentes especializaciones y niveles asistenciales, hace que sea difícil para el estudiante orientarse entre tanta diversidad. Cuanto más pronto conozca el alumno las diferentes opciones profesionales que el futuro podría brindarle, más tiempo tendrá para dirigir sus intereses y sentar las bases de su futura vocación o especialidad en los años siguientes [17].

\section{Bibliografía}

1. Dornan T. Osler, Flexner, apprenticeship, and 'the new medical education'. J R Soc Med 2005; 98: 91-5.

2. Yardley S, Brosnan C, Richardson J. The consequences of authentic early experience for medical students: creation of metis. Med Educ 2013; 47: 109-9.

3. Ster MP. Teaching communication at the Medical School of Ljubljana. Acta Med Acad 2012; 41: 38-46.

4. Elestein M, Forbes JA. Early medical contact. BMJ 1976; 2: 97-8.
5. Hopayian K, Howe A, Dagley V. A survey of UK medical schools' arrangements for early patient contact. Med Teach 2007; 29: 806-13.

6. Dornand T, Littlewood S, Margolis SA, Scherpbier A, Spencer J, Ypinazar V. How can experience in clinical and community settings contribute to early medical education? A BEME systematic review. Med Teach 2006; 28: 13-8.

7. Yardley S, Littlewood S, Margolis SA, Scherpbier A, Spencer J, Ypinazar V,et al. What has changed in the evidence for early experience. Med Teach 2010; 32: 740-6.

8. General Medical Council. Tomorrow's Doctors. Outcomes and standards for undergraduate medical education. London: GMC; 2009.

9. Universitat Pompeu Fabra. Plan de estudios del Grado de Medicina UAB-UPF. URL: http://www.upf.edu/biomed/medicina/ pla. [03.01.2014]

10. Universitat Autònoma de Barcelona. Plan de estudios del Grado de Medicina UAB-UPF. URL: http://www.uab.es/ servlet/Satellite/estudiar/tots-els-estudis/informacio-general/ medicina-grau-uab/upf-1089963374371.html?param1=1216 620146538\&param $11=6$. [03.01.2014].

11. Baños JE, Sentí M, Miralles R. Contacto precoz con la realidad asistencial: una experiencia piloto en medicina. Educ Med 2011; 14: 39-47.

12. Dornan T, Bundy C. What can experience add to early medical education? Consensus survey. BMJ 2004; 329: 834-7.

13. Smithson S, Hart J, Wass V. Students' hopes and fears about early patient contact: lessons to be learned about preparing and supporting students during the first year. Med Teach 2010; 23: e24-30.

14. Iwata K, Gill D. Learning through work: clinical shadowing of junior doctors by first year medical students. Med Teach 2013; 35: 633-8.

15. Johnson AK, Scott CS. Relationship between early clinical exposure and first-year students' attitudes toward medical education. Acad Med 1998; 73: 430-2.

16. Littlewood S, Ypinazar V, Margolis S, Scherpbier A, Spencer S, Dornan T. Early practical experience and the social responsiveness of clinical education: systematic review. BMJ 2005; 331: 387-91.

17. Briggs-Style C, Maxwell JA, Moore GT. The effects of early patient contact: the student's perspective. Acad Med 1990; 65 (Suppl): S33-4. 